\title{
Thermal Analysis of Die Formed Elbow Joint and Mitered Elbow Joint Using Altair HyperWorks Software
}

\author{
Ankur Kumar Pal ${ }^{1}$, Sanjeev Kumar ${ }^{2}$, Ravi Kumar ${ }^{3}$ \\ ${ }^{1}$ (Mechanical Engineering Department, Bipin Tripathi Kumaon Institute of Technology, Dwarahat, Almora, \\ Uttarakhand, India) \\ ${ }^{2}$ (Mechanical Engineering Department, Bipin Tripathi Kumaon Institute of Technology, Dwarahat, Almora, \\ Uttarakhand, India) \\ ${ }^{3}$ (Mechanical Engineering Department, Bipin Tripathi Kumaon Institute of Technology, Dwarahat, Almora, \\ Uttarakhand, India)
}

\begin{abstract}
In this work thermal analysis and comparison of die formed elbow joint and mitered elbow joint of circular duct is done using Altair HyperWorks Software. Results were obtained for conduction and convection through the cross section of elbow joints of the hollow duct. The inner surface of both the duct joints is maintained at constant temperature and ambient air is at certain temperature that is less than inner surface temperature of the duct. Due to temperature difference heat will flow from higher temperature to lower temperature. The material of pipe provides conductive resistance and the surrounding air provides convective resistance. The heat transfer is one dimensional and properties are considered to be isotropic. The duct joints are assumed to be made of steel having known thermal conductivity and density. The surroundings of joints have known convective heat transfer coefficient and temperature. The modelling and meshing is done in Hypermesh, analysis is done in Radioss and results are obtained in hyperview which is a postprocessor in Altair HyperWorks. The different characteristics can be obtained by varying the material of the joints.
\end{abstract}

Keywords:Elbow Joint, Duct, Mitered, Die formed, Altair HyperWorks

\section{Introduction}

The subject of heat transfer and fluid flow through the duct joints from a fundamental viewpoint has been virtually neglected in the literature. This probably resulted from the industrial practice of generally discarding the importance of heat transfer and fluid flow in duct joints in heat transfer equipment. With the advent of nuclear engineering, advancements in HVAC systems and the resulting unconventional heat transfer design problems the increasing industrial use of various types of duct joints in heat exchangers, the problem becomes more than just an academic question.

\section{Literature Review}

Heat conduction is increasingly important in various areas involving thermal analysis. A common example of heat conduction is heating an object in an oven or furnace. An analysis for mix mode of heat transfer using Altair HyperWorks has been demonstrated which gives the contour plots for temperature gradient and flux for a cylinder. The results are useful in finding the various thermal properties at particular location or coordinates[1]. The material remains stationary throughout, neglecting thermal expansion, as the heat diffuses inward to increase its temperature. The importance of such conditions leads to analyze the temperature field by employing sophisticated mathematical and advanced numerical tools [2]. To compute the heat flux at any location, compute thermal stress, expansion, deflection, design insulation thickness, heat treatment method, one need to know temperature field [3]. The problem is known and analytical solution is obtained but the on Altair software and making thermal analysis on it is a new challenge. The conduction of heat on material is by Fourier law and in the fluid around pipe the heat transfer is due to convection. The heat transfer in cylindrical case is of logarithmic variation in contrast to the linear variation in plane wall [4]. In steady one dimensional conduction in cylinder temperature is a function of only radial co-ordinates. The analysis of steady heat flow across a plane wall is simple because the flow area do not change in the flow direction. In the case of cylinder the area for heat flow changes in the direction of heat flow [5]. The flow may be laminar or turbulent which depends upon the Reynolds number. Heat transfer rate tends to be much higher in turbulent flow than in laminar flow. The rate of heat transfer by convection is usually function of surface area, geometry and thermo physical properties [6].

\section{Objective Of Present Work}

Effort has been made to predict the heat flux on the outer surface of the elbow joints under consideration. Subsequently, the type of joint can be selected as per the applications either heat transfer maximization or minimization. 
4.1 Heat conduction through circular duct

\section{Mathematical Modelling}

Governing Equation

Assumptions

1. One dimensional steady state heat transfer.

2. Constant thermal conductivity of material.

3.No heat generation in the material.

4.The heat transfer coefficient between the duct joint and the surrounding fluid, i.e., air is constant.

5.Heat transfer by radiation is negligible.

Governing differential equation for the heat transfer through circular duct is as follows:

Fig. 1 shows a circular duct of inside radius $r_{1}$, outside radius $r_{2}$, length $L$, and thermal conductivity k. The inside surface is maintained at constant temperatures $T_{1}$. Ambient temperature is $T_{\infty}=25^{\circ} \mathrm{C}$. The convective heat transfer coefficient of surroundings is $\mathrm{h}$.

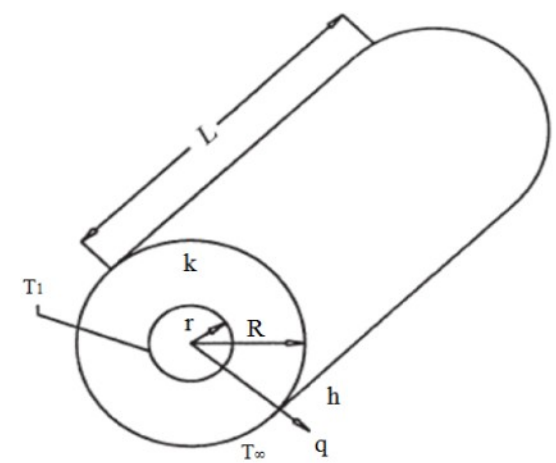

Fig.1Heat transfer through a circular duct

For steady-state conduction in the radial direction with no internal heat generation and constant thermal conductivity, the general heat conduction equation is as follows:

Boundary Conditions

$$
\frac{\mathrm{d}}{\mathrm{dr}}\left(\mathrm{r} \frac{\mathrm{dT}}{\mathrm{dr}}\right)=0
$$

(a) The temperature at the inner surface of the duct, i.e., at $40 \mathrm{~mm}$ radius is $60^{\circ} \mathrm{C}$.

Mathematically: $\mathrm{T}(\mathrm{r}=40 \mathrm{~mm})=60^{\circ} \mathrm{C}$

(b) The ambient temperature is $25^{\circ} \mathrm{C}$.

Mathematically: $\mathrm{q}_{\mathrm{cond}}(\mathrm{r}=50 \mathrm{~mm})=\mathrm{hdT}$

\subsection{Thermal Resistance Concept}

The thermal resistance can be expressed as electrical analogy given as:

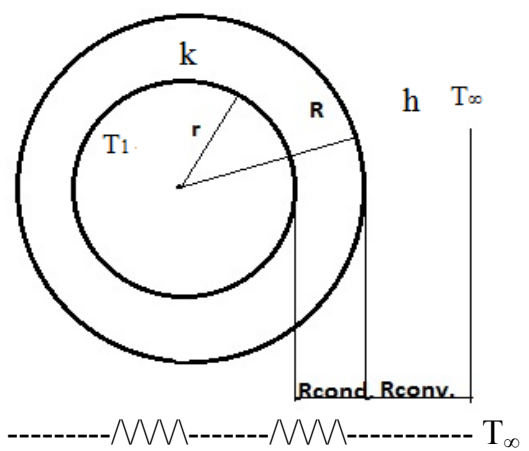

Fig.2Conductive and Convective resistance

Fig. 2 shows a circular duct cross section of inside radius $r_{1}$, outside radius $r_{2}$ and thermal conductivity $\mathrm{k}$. The inside surface is maintained at constant temperatures $\mathrm{T}_{1}$. Ambient temperature is $\mathrm{T}_{\infty}=25^{\circ} \mathrm{C}$. The convective heat transfer coefficient of surroundings is h. The overall heat transfer is given by: 


$$
\mathrm{Q}=\frac{\Delta \text { Toverall }}{\Sigma \text { Rth }}
$$

Where Rth is total thermal resistance and is sum of conductive and convective resistance.

Where:

$$
\sum \mathrm{Rth}=\mathrm{R} \operatorname{cond}+\mathrm{R} \operatorname{conv}(3)
$$

Rate of heat transfer is given by:

$$
\begin{aligned}
\text { Rcond } & =\frac{\ln (\mathrm{R} / \mathrm{r})}{2 \pi \mathrm{kL}} \\
\text { Rconv } & =\frac{1}{2 \pi \mathrm{RLh}}
\end{aligned}
$$

$$
Q=\frac{2 L\left(T_{1}-T_{2}\right)}{\left(\frac{\ln \left(\frac{R}{r}\right)}{k}+\frac{1}{h R}\right)}
$$

\section{Problem Formulation}

In the present work thermal analysis is done for three standard elbow joints of circular duct. All the elbow joints considered in the work are $90^{\circ}$ joints and have same dimensions for cross section and material thickness. The material of the joints is stainless steel. In this case, the inner surface of the joint is constant at $60^{\circ} \mathrm{C}$ due to flow of hot fluid. The ambient temperature is $25^{\circ} \mathrm{C}$. The direction heat flow will be from the inner surface towards the surroundings. The material and the surroundings will provide the resistance to heat flow.

The duct joints considered for analysis are

(a) Die formed elbow

(b) Mitered elbow

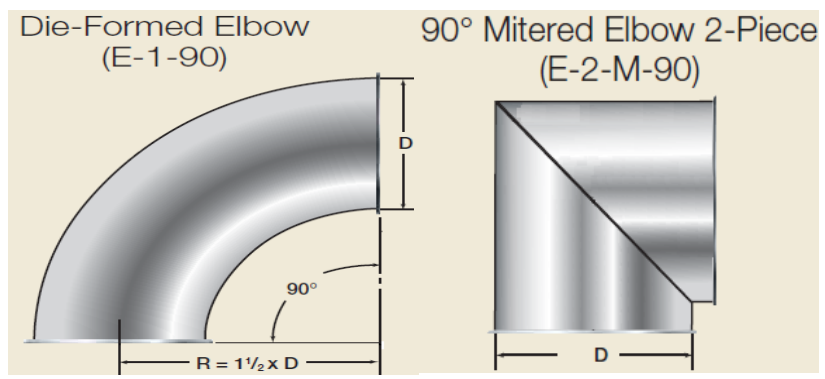

Fig.3Types of $90^{\circ}$ Elbow Joints

Dimensions:

Inner Diameter(d): $\quad 80 \mathrm{~mm}$

Outer Diameter(D): $\quad 100 \mathrm{~mm}$

Elbow angle: $\quad 90^{\circ}$

Elbow Radius(R): $\quad 150 \mathrm{~mm}$

Table 1 Properties used in analysis

\begin{tabular}{|l|l|l|}
\hline 1. & Material of joints & Stainless Steel \\
\hline 2. & Density $(\rho)$ & $7900 \mathrm{~kg} / \mathrm{m}^{3}$ \\
\hline 3. & Thermal conductivity(k) & $14.9 \mathrm{~W} / \mathrm{m} . \mathrm{K}$ \\
\hline 4. & Coefficient of thermal expansion $(\alpha)$ & $17.2 \times 10^{-6} / \mathrm{K}$ \\
\hline 5. & Poisson's Ratio $(\mu)$ & 0.30 \\
\hline 6. & Young's Modulus(E) & $210 \mathrm{GPa}$ \\
\hline 7. & Convective Heat Transfer Coefficient $(\mathrm{h})$ & $20 \mathrm{~W} / \mathrm{m}^{2} . \mathrm{K}$ \\
\hline
\end{tabular}

The modelling and meshing of the joints according to the dimensions mentioned is done in Hypermesh. The thermal analysis is done in Radioss. The unit system used for analysis in RADIOSS is millimetre-secondtonne(mm-s-t).In this system the length, time and mass are considered in millimetre, seconds and tonnes respectively. So, units of flux in the results is $\mathrm{kW} / \mathrm{m}^{2}$.

\section{About Altair Hyperworks}

Altair HyperWorks is the engineering framework for product design for optimization of product performance, automating design process and improving profitability with an open and flexible environment. HyperWorks comprises a suite of application that includes Hypermesh, Optistruct, Radioss, Hypercrash, Hyperview. Hypergraph 3D, Motionview, Mediaview, Textview, FM Model, Hyperstudy, Motionsolve, Batchmesher, Manufacturing Solution Hyperplayer, Altair Data Manager, Assembler process manager, 
providing modelling and assembly, robust design and optimization, design analysis, visualization and reporting, virtual manufacturing and Automation and data management.

\subsection{Modelling And Meshing In HyperMesh}

\section{Procedure}

The modelling of the elbow joints is done in Hypermesh as per the dimensions mentioned in previous section. The meshing of the models is also done in Hypermesh. The element density for all the joints is taken same, i.e., 40. Firstly, a 2D mesh is generated. Taking it as a reference the final 3D mesh is generated. It is also important to delete every other geometries and elements by masking the 3D mesh because they can create hurdles during the thermal analysis in radioss. The final meshed joints are shown below:
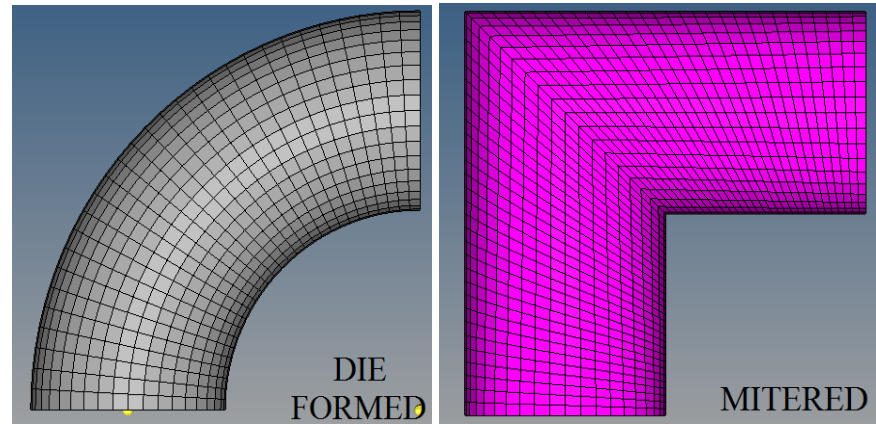

Fig.4 Meshed Elbow Joints

\subsection{Analysis in RADIOSS}

Steps in given problem

- Create the thermal material and property

- Create and apply the thermal boundary conditions on the model

- Submit the job to RADIOSS

- Post-process the results in HyperView

Step 1: Load the RADIOSS (Bulk Data) User Profile and draw the model.

Step 2: Create the thermal material properties. Create the material and property collectors before creating the component collectors.

Step 3: Link the material and property to the existing structure. Once the material and property are defined, they need to be linked to the structure.

Step 4: Create temperatures on the inner surface of the duct joint.

Step 5: Create ambient temperature.

Step 6: Create CHBDYE surface elements for heat convection. Surface elements are to be created to simulate the heat exchange between the solid pipe and the surrounding air.

Step 7: Define the convection boundary condition to surface elements

Step 8: Create a heat transfer load step. A RADIOSS steady state heat convection load step will be created which references the thermal boundary conditions in the load collector spc temp. The gradient, flux, and temperature output for the heat transfer analysis will also be requested in the load steps panel.

Step 9: Launch RADIOSS View and Post process the Results. Gradient temperatures and flux contour results for the steady state heat conduction analysis are computed from RADIOSS. Post processing of the results is done in Hyper View.
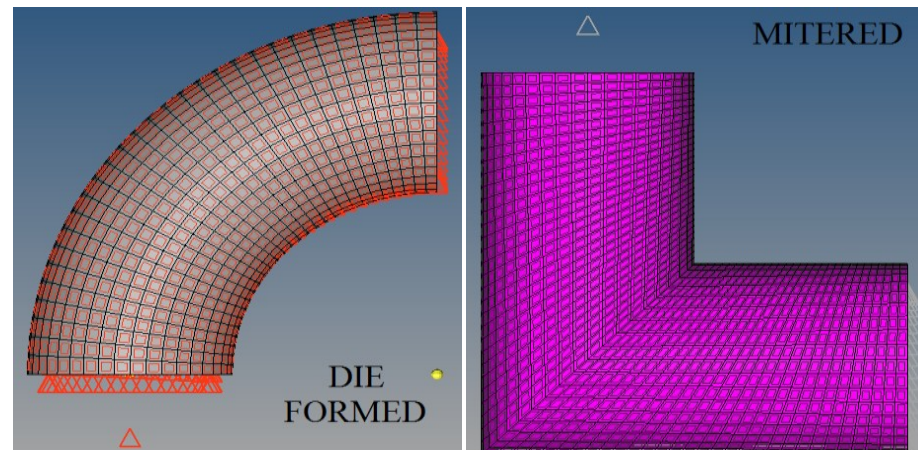

Fig.5The elbow joints after applying the loads and boundary conditions 
Results are viewed in HyperView.

\section{Results And Discussion}

\subsection{Contour Plots for Flux}

(a) Die Formed Elbow

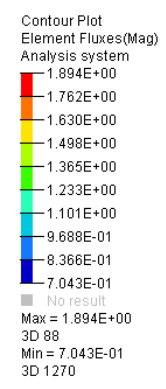

$\sum_{-X}^{Z}$
Model info: C: : final projectlatresults $\backslash E-1-90 \backslash E-1-90 . h 3 d$

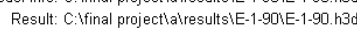
Subcase 1 (heat_transfer) : Heat Transfer Analysis
Frame 4

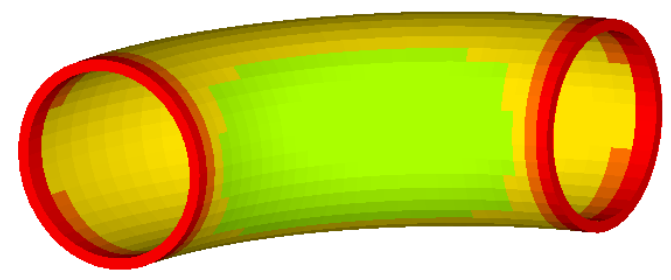

Fig.6Die Formed Elbow

The maximum heat flux on the outer surface of die formed elbow ranges from 1.498 to 1.630 units. Therefore the average maximum heat flux is 1.564 units, i.e., $1564 \mathrm{~W} / \mathrm{m}^{2}$.

(b) Mitered Elbow
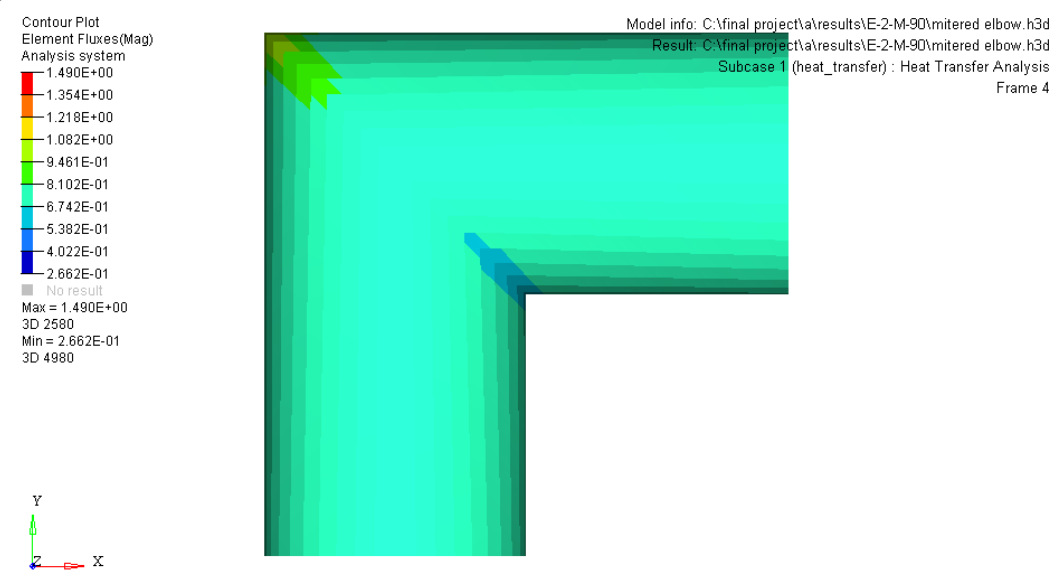

Fig.7 Mitered Elbow

The maximum heat flux on the outer surface of mitered elbow ranges from 0.6742 to 0.8102 units. Therefore the average maximum heat flux is 0.7422 units, i.e., $742.2 \mathrm{~W} / \mathrm{m}^{2}$.

\subsection{Graph}

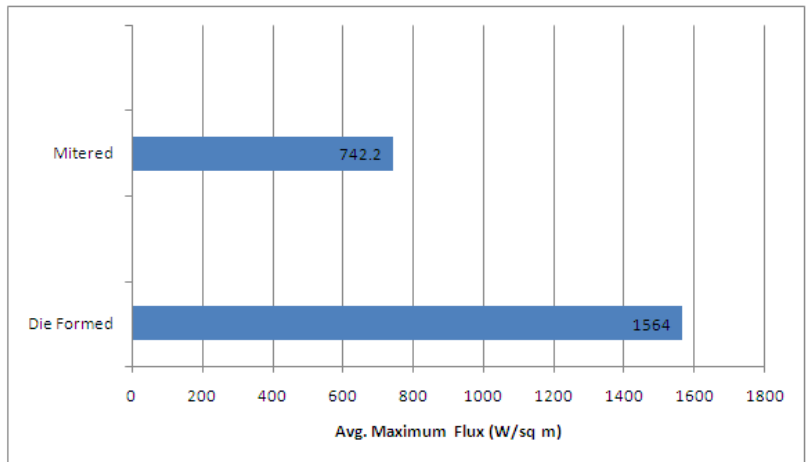

Graph 6.1 Max. Flux(avg.) in outer surface 


\section{Conclusion}

The maximum heat flux on the outer surface is available in the die formed elbow joint. Thus, it can be used in applications where maximization of heat transfer is desired.

The minimum heat flux on the outer surface is available in the mitered elbow joint. Thus, it can be used in applications where minimization of heat transfer is desired.

\section{References}

[1]. P. Bhatt and A. Gupta, Thermal Analysis of Hollow Pipe Using Altair HyperWorks, International Journal of Engineering Research \& Technology 6(1), 2012

[2]. P.J. Schneider, Conduction Heat transfer(Addison Wesley, 1957).

[3]. E.Fried,Thermal conduction contribution to heat transfer at contacts, in Thermal Conductivity, Vol. 2 R.P. Tye (Ed.),(London: Academic, 1969) 24-33.

[4]. V. Gnielinski, New Equations for Heat And Mass Transfer in Pipe and Channel Flow (Heat Transfer: Soviet Research, 1973)

[5]. Frank P. Incropera and David P. Dewitt, Fundamentals of Heat and Mass Transfer (Wiley Publication, Fifth Edition,2009).

[6]. Milles A.F and Ganesan V, Heat and Mass Transfer(Pearson Education, Second Edition, 2009).

[7]. ISOVER, HVAC Ducts Handbook, 2012.

[8]. Materials Data Book (Cambridge University Engineering Department, 2003 ed.) 